\title{
A SUPERLINEARLY CONVERGENT MINIMAX ALGORITHM FOR MICROWAVE CIRCUIT DESIGN
}

\author{
J.W. Bandler ${ }^{*}$, W. Kellermann ${ }^{*}$ and K. Madsen ${ }^{* * *}$ \\ * Simulation Optimization Systems Research Laboratory \\ and Department of Electrical and Computer Engineering, \\ McMaster University, Hamilton, Canada L8S 4L7 \\ *** Institute for Numerical Analysis, \\ Technical University of Denmark \\ DK-2800 Lyngby, Denmark
}

\begin{abstract}
A new and highly efficient algorithm for nonlinear minimax optimization is presented. A novel approach to worstcase design of microwave circuits using the present algorithm is proposed. The algorithm is proved through practical design of multiplexers, involving up to 75 design variables.

\section{INTRODUCTION}

The algorithm of this paper, based on the work of Hald and Madsen [1], combines linear programming methods with quasi-Newton methods and has sure convergence properties.

A novel approach to worst-case tolerance design of microwave circuits taking full advantage of the present algorithm is proposed. The approach, which is believed to be new to the microwave tolerance design area, integrates a search technique for maxima of the response (a technique based on cubic interpolation) with the worst-case search using linearly constrained optimization.

An optimization procedure for practical design of contiguous and noncontiguous-band microwave multiplexers using the present algorithm is described and illustrated by a 5 channel, $11 \mathrm{GHIz}$ multiplexer design.
\end{abstract}

\section{DESCRIPTION OF THE ALGORITHM}

Let $\mathrm{f}_{\mathrm{j}}(\mathbf{x}) \triangleq \mathrm{f}_{\mathrm{j}}\left(\mathrm{x}_{1}, \ldots, \mathrm{x}_{\mathrm{n}}\right), j=1, \ldots, \mathrm{m}$, be a set of $\mathrm{m}$ nonlinear, continuously differentiable functions and $\mathbf{x} \triangleq \mid l l l_{1} \quad x_{2}$ $\left.\ldots x_{n}\right|^{\mathrm{T}}$ a set of $n$ parameters to be optimized.

We consider the optimization problem

$$
\operatorname{minimize} \mathbf{F}(\mathbf{x}) \triangleq \max \left\{\mathrm{f}_{\mathbf{j}}(\mathbf{x})\right\}
$$

$$
\mathbf{x} \quad \mathrm{j}
$$

subject to

$$
\begin{array}{ll}
\mathbf{a}_{1}^{\mathrm{T}} \mathbf{x}+\mathrm{b}_{\mathrm{i}}=0, & \mathrm{i}=\mathbf{1}, \ldots, \mathrm{t}^{\prime}, \\
\mathbf{a}_{\mathrm{i}}^{\mathrm{T}} \mathbf{\mathrm { T }}+\mathrm{b}_{\mathrm{i}} \geq 0, & \mathrm{i}=\left(\mathrm{t}^{\prime}+1\right), \ldots, \mathrm{t},
\end{array}
$$

where $a_{i}$ and $b_{i}, i=1, \ldots, t$, are constants.

This work was supported in part by the Natural Sciences and Engineering Research Council of Canada under Grant G1135.

+ J.W. Bandler is also with Optimization Systems Associates, 163 Watson's Lane, Dundas, Ontario, Canada L9H $6 \mathrm{~L} 1$.
The algorithm for solving ( 1 ) is a combination of two methods denoted Method 1 and Method 2. Method 1 is intended to be used far away from a solution whereas Method 2 is a local method

Method 1 This is essentially the algorithm of Madsen [2]. At the kth step a feasible approximation $\mathbf{x}_{\mathrm{k}}$ of a solution of (1) and a local bound $\Lambda_{k}$ are given. In order to find a better estimate of a solution the following linearized problem is solved,

$$
\underset{\mathbf{h}}{\operatorname{minimize}} \overrightarrow{\mathrm{F}}\left(\mathbf{x}_{\mathrm{k}}, \mathbf{h}\right) \triangleq \max _{j}\left\{\mathbf{f}_{\mathrm{j}}\left(\mathbf{x}_{\mathrm{k}}\right)+\mathbf{f}_{\mathrm{j}}^{\prime}\left(\mathbf{x}_{\mathrm{k}}\right)^{\mathrm{T}} \mathbf{h}\right\}
$$

subject to

$$
\begin{array}{ll}
\|\mathbf{h}\|_{\infty} \leq \Lambda_{\mathrm{k}} & \\
\mathbf{a}_{\mathrm{i}}^{\mathrm{T}}\left(\mathbf{x}_{\mathbf{k}}+\mathbf{h}\right)+\mathrm{b}_{\mathrm{i}}=0, & \mathrm{i}=1, \ldots, \mathrm{t}^{\prime}, \\
\mathbf{a}_{\mathrm{i}}^{\mathrm{T}}\left(\mathbf{x}_{\mathbf{k}}+\mathbf{h}\right)+\mathrm{b}_{\mathrm{i}} \geq 0, & \mathrm{i}=\left(\mathrm{t}^{\prime}+1\right), \ldots, \mathrm{t},
\end{array}
$$

where $\mathbf{f}^{\prime}(\mathbf{x})$ is the gradient of $\mathbf{f}_{\mathrm{j}}$ w.r.t. $\mathbf{x}$.

The solution of (2), denoted $\mathbf{h}_{k}$, is found by linear programming. The next iterate, $\mathbf{x}_{\mathrm{k}+\mathrm{l}}$, is $\mathbf{x}_{\mathrm{k}}+\mathbf{h}_{\mathrm{k}}$ provided that $\mathrm{F}\left(\mathbf{x}_{\mathrm{k}}+\mathbf{h}_{\mathrm{k}}\right)<\mathrm{F}\left(\mathbf{x}_{\mathrm{k}}\right)$. Otherwise $\mathbf{x}_{\mathrm{k}+1}=\mathbf{x}_{\mathrm{k}}$

The local bound $\Lambda_{k}$ is introduced because the linear model (2) is a good approximation of (1) only in some neighbourhood of $x_{k}$. Therefore, it makes sense to consider only small values of $\|\mathbf{h}\|$ in connection with the linear model (2). The size of the bound is adjusted in every iteration to make sure that the linear approximations are valid.

When the solution is singular [3], the final convergence can be very slow. Fig. 1 shows contours for a 2 -section transmission-line transformer problem with variables $Z_{1}$ and $\mathrm{Z}_{2}$, where the minimax functions correspond to the reflection coefficient. Fig. 2 shows contours for the same two-section transformer problem. The optimization variables are now $\ell_{1} / \ell_{4}$ and $Z_{1}$. Here, the problem is regular and 5 iterations are sufficient to reach the vicinity of the solution

Method 2 It is assumed that a point near a solution $z$ is known, and that the active sets $\mathrm{A}(\mathbf{z}) \triangleq\{\mathrm{j} \mid \mathrm{f}(\mathbf{z})=\mathrm{F}(\mathbf{z})\}$ and $\mathrm{C}(\mathbf{z}) \triangleq$ $\left\{\mathbf{i} \mid \mathbf{a}_{\mathrm{i}}{ }^{\mathrm{T}} \mathbf{z}+\mathrm{b}_{\mathrm{i}}=0\right\}$ are known. At a local minimum $\mathbf{z}$ of (1) the following necessary conditions hold,

$$
\begin{array}{ll}
\sum_{j \in A(z)} \Lambda_{j} f_{j}^{\prime}(z)-\sum_{i \in C(z)} \mu_{i} a_{i}=0, & \\
\sum_{j \in A(z)} \Lambda_{j}-1=0, & \\
f_{j_{0}}(z)-f_{j}(z)=0, & j \in A(z) \backslash\left\{j_{0}\right\}, \\
a_{i}^{T} z+b_{i}=0, & i \in C(z),
\end{array}
$$




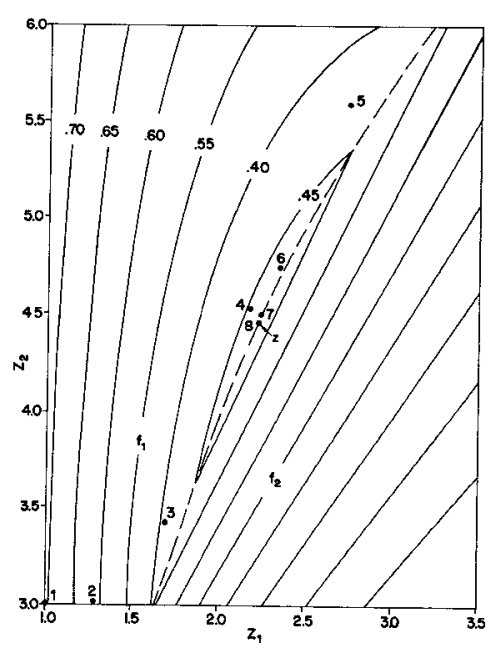

Fig. 1 Two-dimensional singular minimax problem arising from optimization of a two-section transmission-line transformer.

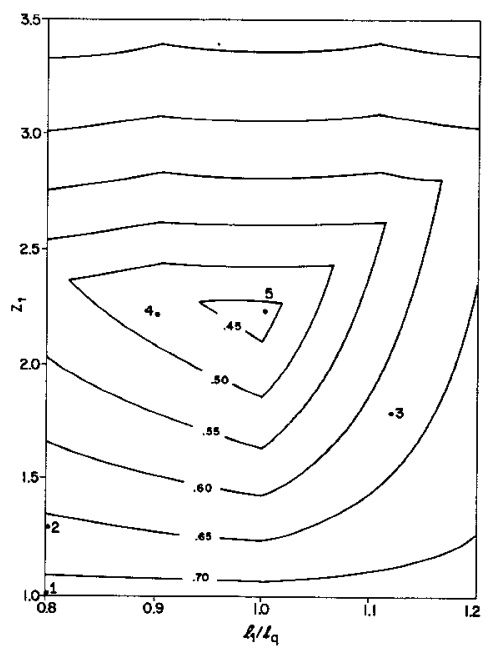

Fig. 2 Two-dimensional regular minimax problem arising from optimization of a two-section transmissionline transformer.

where the multipliers $\lambda_{j}$ and $\mu_{i}$ are nonnegative and $j_{0} \in A(z)$ is fixed. Method 2 is an approximate Newton method for solving the nonlinear system (3) (in the variables $(\mathbf{z}, \boldsymbol{\Lambda}, \boldsymbol{\mu})$ ). Exact first. derivatives are used but the matrix $\Sigma \lambda_{j} f_{j}^{\prime \prime}(z)$ is approximated, where $\mathbf{f}_{j}^{\prime \prime}(\mathbf{z})$ is the Hessian of $f_{j}$ w.r.t. $z$, using a modified BFGS update.

The Combined Method The combined method is the algorithm which we recommend and use in this paper. Initially, Method 1 is used and the active sets used in (3) are estimated. When a singular local minimum seems to be approached a switch to Method 2 is made. If the Method 2 iteration is unsuccessful Method 1 is used again. Several switches between the two methods may take place.

For this paper we have used the MMIC version [4] of the algorithm, based on the earlier implementation due to Hald [5].

\section{COMPARISON WITH OTHER ALGORITHMS}

Table I shows the performance of the new algorithm as compared to other algorithms using a 3-section transmissionline transformer as a test problem [6]. T'wo starting points have been used, $x_{0}{ }^{l}=\left[\begin{array}{llllll}0.8 & 1.5 & 1.2 & 3.0 & 0.8 & 6.0\end{array}\right]^{\mathrm{T}}$ and $\mathbf{x}_{0}{ }^{2}=$ $\left[\begin{array}{llllll}1.0 & 1.0 & 1.0 & 3.16228 & 1.0 & 10.0\end{array}\right]^{\mathrm{T}}$. 'Table I also shows results obtained using the present algorithm with a cubic interpolation search for maxima of the response. Using this technique the number of sample points can be reduced from 11 to 4 and we do not have to know in advance the location of frequency points corresponding to the maxima of the response.

\section{WORST-CASE NETWORK DESIGN}

In this section we formulate the fixed tolerance problem (FTP) $[11,12]$ embodying worst-case search and the selection of sample points for the discrete approximation of a continuous problem.

A design consists of design data of the nominal point $\mathbf{x}^{0}$ $\triangleq\left|x^{0} x_{2}{ }^{0} \ldots x_{n}{ }^{0}\right|^{T}$ and a set of associated tolerances $\varepsilon \triangleq\left[\varepsilon_{1} \varepsilon_{2} \ldots\right.$ $\left.\varepsilon_{n}\right]^{T}$, where $n$ is the number of network parameters [11]. An outcome of a circuit is any point $x \triangleq\left[\begin{array}{llll}x_{1} & x_{2} & \ldots & x_{n}\end{array}\right]^{\mathrm{T}}$ such that $x_{i}=$ $x_{1}{ }^{0}+\varepsilon_{i} \mu_{i},-1 \leq \mu_{i} \leq 1, i=1,2, \ldots, n$. The tolerance region $R_{c}$ is defined as $R_{\varepsilon} \triangleq\left\{x \mid x_{1}=x_{i}{ }^{0}+\varepsilon_{i} \mu_{i}, i=1,2, \ldots, n\right\}$. The extreme points of $R_{c}$ are called the vertices and are obtained by setting $\mu_{\mathrm{i}}= \pm 1$.

We consider a set of $m$ nonlinear functions

$\mathrm{f}_{\mathrm{j}}\left(\mathrm{x}^{0}\right) \triangleq \mathrm{f}\left(\mathrm{x}^{0}, \omega_{\mathrm{j}}\right), \mathrm{j} \in \mathrm{J} \triangleq\{1,2, \ldots, \mathrm{m}\}$,

where $\omega_{j}, j \in J$, is an independent parameter (frequency). The number of functions $m$ is equal to

$$
\mathrm{m}=\mathrm{m}_{\max }+2 \text {, }
$$

where $m_{\max }$ is the number of the maxima of the response and 2 represents the edges of the frequency interval $\left[\omega_{\rho}, \omega_{u} \mid\right.$.

The fixed tolerance problem can he defined on the basis of the worst-case objective function [12] as that of determining

$$
\begin{array}{ll}
\min F\left(x^{0}\right)= & \min \max \left\{\max f_{j}(x)\right\} . \\
x^{0} & x^{0} \quad j \in J \quad x \in R_{\varepsilon}
\end{array}
$$

For each outer iteration of minimization w.r.t. $x^{0} \mathrm{~m}$ frequency points are determined (by a search technique based on cubic interpolation) and $m$ linearly constrained optimizations are performed to find the worst cases.

At the kth outer iteration of minimization we have an approximation $x_{k}{ }^{0}$ of the solution and we solve $m$ linearly constrained optimizations, where the $j$ th problem, $j \in J$, is

$$
\operatorname{minimize}\left(-f_{j}\left(x_{k}\right)\right)
$$

subject to

$$
\left(x_{i}^{0}\right)_{k}-\varepsilon_{i} \leq\left(x_{i}\right)_{k} \leq\left(x_{i}^{0}\right)_{k}+\varepsilon_{i}, \quad i=1,2, \ldots, n .
$$

The 3-section transmission-line transformer is used to illustrate the approach and its validity for worst-case design Numerical results are summarized in Table II.

\section{CONTIGUOUS AND NONCONTIGUOUS BAND MULTIPLEXER DESIGN}

Practical design and manufacture of contiguous and noncontiguous band multiplexers consisting of multi-cavity filters distributed along a waveguide manifold has been a problem of significant interest $[13,14]$. 
TABLE I

COMPARISON OF ALGORITHMS FOR THE 3-SECTION TRANSFORMER (NUMBER OF FUNCTION EVALUATIONS)

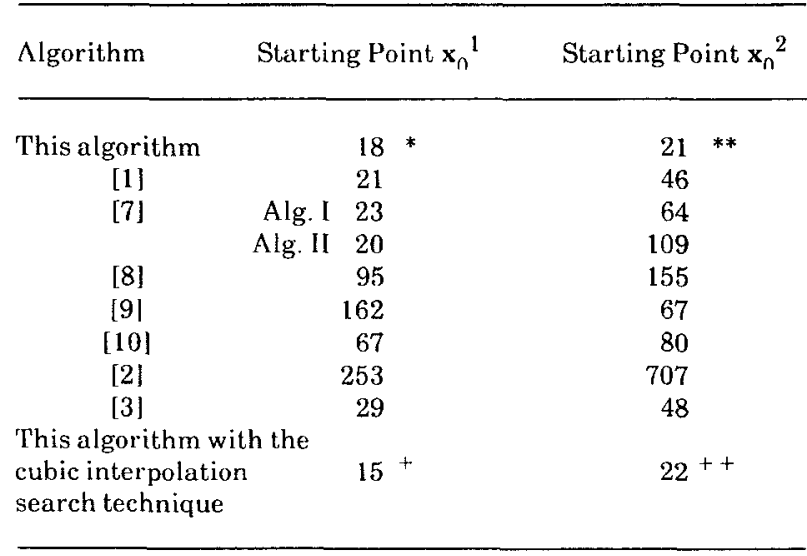

Execution times on Cyber $170 / 815$ in seconds are ${ }^{*} 0.6,{ }^{* *} 0.7$, ${ }^{+} 0.57$ and ${ }^{+}+0.85$

"Active" frequency points selected by the cubic interpolation search are $0.50000,0.76999,1.23001$, and 1.50000

We present here a general multiplexer optimization procedure exploiting exact network sensitivities. The simulation and sensitivity analysis aspect of the problem has been described in [15]. All design parameters of interest, e.g., waveguide spacings, input-output and filter coupling parameters, can be directly optimized.

A wide range of possible multiplexer optimization problems can be formulated and solved by appropriately defining specifications on common port return loss and individual channel insertion loss functions. The minimax error functions are created using those specifications, simulated exact multiplexer responses and weighting factors

The procedure is illustrated by designing an $11 \mathrm{GHz}, 5$ channel multiplexer having the center frequencies and bandwidths (similar to those of $|14|$ ) given in Table III

We start the design process with five identical six-pole, pseudo-elliptic function filters. Starting values of the coupling coefficients for the filters are taken from $\{13\}$. The initial spacing lengths are set equal to $\lambda_{4 k} / 2$ (half the wavelength corresponding to the $k$ th center frequency).

Fig. 3 shows the responses of the multiplexer at the start of the optimization process.

For five channels a total of 75 parameters are optimized including spacings, input-output transformer ratios, cavity resonances and coupling coefficients. The filters are assumed lossy and dispersive, and waveguide junctions are assumed nonideal. The results of optimization are shown in Fig. 4. Typical running times are from a few seconds to a few minutes on a Cyber 170/815 computer depending on the size of the problem.

\section{CONCLUSIONS}

We have described a new and highly efficient algorithm
TABLE II

\section{FIXED TOLERANCE PROBLEM FOR THE} THREE-SECTION 10:1 TRANSFORMER

\begin{tabular}{lc}
\hline & 4 \\
Number of Minimax Functions & 6 \\
Number of Variables & $10^{-5}$ \\
Required Accuracy of the Solution & $5 \%$ \\
Assumed Tolerances & \\
Step Size in the Cubic & 0.1 \\
Interpolation Search & \\
Solution Vector $\quad \ell_{1} / \ell_{\mathrm{q}}=0.96373$ & $\mathrm{Z}_{2}=3.22493$ \\
$\quad \mathrm{Z}_{1}=1.67797$ & $\ell_{3} / \ell_{\mathrm{q}}=0.96483$ \\
$\quad \ell_{2} / \ell_{\mathrm{q}}=0.98720$ & $\mathrm{Z}_{3}=6.04817$ \\
"Nctive" Frequency Points 0.50000, & $0.78726,1.27242$, \\
1.50000 & \\
Maximum Refl. Coefficient & 0.33589 \\
Number of Function Evaluations & 32 \\
Execution Time on Cyber $170 / 815$ & 8.1 \\
(in seconds)
\end{tabular}

TABLE III

MULTIPLEXER CENTER FREQUENCIES AND BANDWIDTHS

\begin{tabular}{ccc}
\hline Channel & $\begin{array}{c}\text { Center Frequency } \\
\text { (MHz) }\end{array}$ & $\begin{array}{c}\text { Bandwidth } \\
(\mathrm{MHz})\end{array}$ \\
\hline 1 & 10992.5 & 81 \\
2 & 11075.0 & 76 \\
3 & 11155.0 & 76 \\
4 & 11495.0 & 76 \\
5 & 11618.5 & 154 \\
\hline
\end{tabular}

for nonlinear minimax optimization problems which arise in microwave circuit design. The algorithm combines linear programming methods with quasi-Newton methods and the convergence is at least superlinear.

We have presented a novel approach to worst-case tolerance design of microwave circuits integrating a cubic interpolation based search technique for maxima of the response with the worst-case search using linearly constrained optimization.

The robustness of the algorithm presented makes possible practical design of contiguous and noncontiguous band microwave multiplexers.

\section{ACKNOWLEDGEMENTS}

The authors are grateful to S.H. Chen, S. Daijavad, M.L. Renault and W.M. Zuberek for their high level contribution to this research program. 


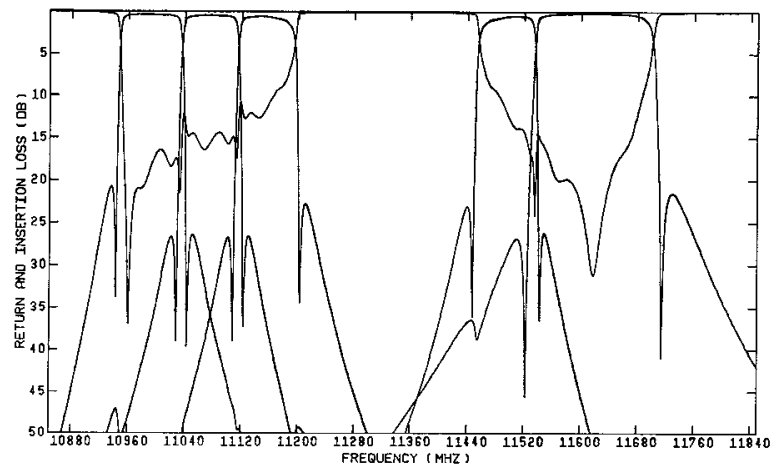

Fig. 3 Responses of the 5-channel, $11 \mathrm{GHz}$ multiplexer at the starting point.

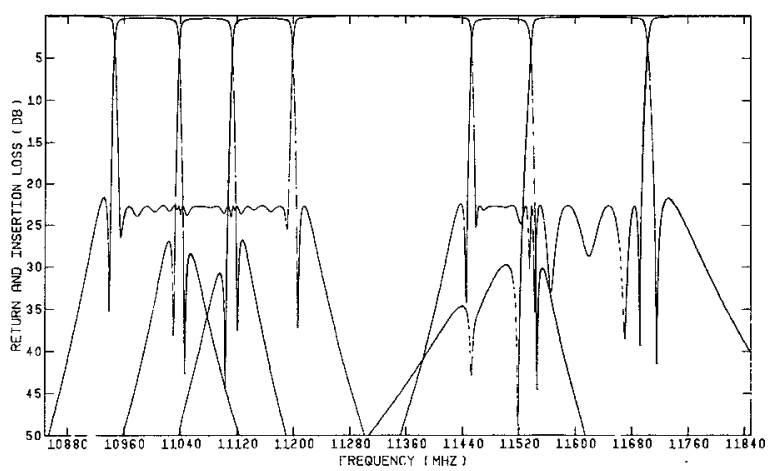

Fig. 4 The optimized responses of the 5-channel, $11 \mathrm{GHz}$ multiplexer.

\section{REFERENCES}

[1] J. IIald and K. Madsen, "Combined LP and quasiNewton methods for minimax oplimization", Mathematical Programming, vol. 20, 1981, pp. 49-62.

[2] K. Madsen, "An algorithm for minimax solution of overdetermined systems of nonlinear equations", J.

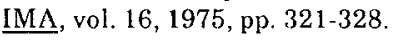

[3] K. Madsen and H. Schjaer-Jacobsen, "Singularities in minimax optimization of networks", IEEE Trans. Circuits and Systems, vol. CAS-23, 1976, pp. 456- 460.
J.W. Bandler and W.M. Zuberek, "MMLC - a Fortran package for linearly constrained minimax optimization", Department of Electrical and Computer Engineering, McMaster University, Hamilton, Canada, Report SOS-82-5, 1982.

[5] J. Hald, "MMLA1Q, a Fortran subroutine for linearly constrained minimax optimization", Report no. NI-8101, Institute for Numerical Analysis, Tech. Univ. of Denmark, 1981

[6] J.W. Bandler and P.A. Macdonald, "Optimization of microwave networks by razor search", IEEE Trans. Microwave Theory Tech., vol. MTT-17, 1969 , pp. 552562 .

[7] D. Agnew, "Improved minimax optimization for circuit design", [EEE Trans. Circuits and Systems, vol. CAS28,1981 , pp. $791-803$.

[8] J.W. Bandler and C. Charalambous, "New algorithms for network optimization", IEEF Trans. Microwave Theory Tech., vol. MT"T-21, 1973, pp. 815-818.

[9] C. Charalambous and A.R. Conn, "Optimization of microwave networks", IEEE 'Trans. Microwave Theory Tech., vol. M'TT-23, 1975, pp. 834-838.

[10] A.R. Conn, "An efficient second order method to solve the (constrained) minimax problem", Dept. of Combinatorics and Optimization, University of Waterloo, Waterloo, Ontario, Report CORR 79-5, 1979.

[11] J.W. Bandler, P.C. Liu and H. Tromp, "A nonlinear programming approach to optimal design centering, tolerancing and tuning", IEEE Trans. Circuits and Systems, vol. CAS-23, 1976, pp. 155-165

[12] H. Schjaer-Jacobsen and K. Madsen, "Algorithms for worst-case tolerance optimization", IEEE Trans. Circuits and Systems, vol. CAS-26, 1979, pp. 775- 783.

[13] M.H. Chen, "A 12-channel contiguous band multiplexer at KU-band", 1983 IEEE Int. Microwave Symp. Digest (Boston, 1983), pp. 77-79.

[14] R.G. Egri, A.E. Williams and A E. Atia, "A contiguousband multiplexer design", 1983 IEEE Int. Microwave Symp. Digest (Boston, 1983), pp. 86-88.

[15] J.W. Bandler, S.H. Chen, S. Daijavad and W. Kellermann, "Optimal design of multi-cavity filters and contiguous-band multiplexers", Proc, 14th European Microwave Conference (Liege, Belgium, 1984), pp. 863-868. 